University of New Hampshire

University of New Hampshire Scholars' Repository

Physics Scholarship

Physics

$10-1-2010$

\title{
GCR access to the Moon as measured by the CRaTER instrument on LRO
}

A. W. Case

Harlan E. Spence

University of New Hampshire, harlan.spence@unh.edu

M. J. Golightly

University of New Hampshire

J. C. Kasper

J. B. Blake

See next page for additional authors

Follow this and additional works at: https://scholars.unh.edu/physics_facpub

Part of the Physics Commons

\section{Recommended Citation}

Case, A. W., H. E. Spence, M. J. Golightly, J. C. Kasper, J. B. Blake, J. E. Mazur, L. W. Townsend, andC. J. Zeitlin (2010), GCR access to the Moon as measured by the CRaTER instrument on LRO,Geophys. Res. Lett., 37, L19101, doi:10.1029/2010GL045118.

This Article is brought to you for free and open access by the Physics at University of New Hampshire Scholars' Repository. It has been accepted for inclusion in Physics Scholarship by an authorized administrator of University of New Hampshire Scholars' Repository. For more information, please contact Scholarly.Communication@unh.edu. 


\section{Authors}

A. W. Case, Harlan E. Spence, M. J. Golightly, J. C. Kasper, J. B. Blake, J. E. Mazur, L. W. Townsend, and C. J. Zeitlin 


\title{
GCR access to the Moon as measured by the CRaTER instrument on LRO
}

\author{
A. W. Case, ${ }^{1}$ H. E. Spence, ${ }^{1,2}$ M. J. Golightly ${ }^{2}$ J. C. Kasper, ${ }^{3}$ J. B. Blake, ${ }^{4}$ J. E. Mazur, ${ }^{5}$ \\ L. W. Townsend, ${ }^{6}$ and C. J. Zeitlin ${ }^{7}$ \\ Received 20 August 2010; accepted 27 August 2010; published 2 October 2010.
}

[1] Recent modeling efforts have yielded varying and conflicting results regarding the possibility that Earth's magnetosphere is able to shield energetic particles of $>10 \mathrm{MeV}$ at lunar distances. This population of particles consists of galactic cosmic rays as well as energetic particles that are accelerated by solar flares and coronal mass ejections. The Cosmic Ray Telescope for the Effects of Radiation (CRaTER) onboard the Lunar Reconnaissance Orbiter is in orbit about the Moon and is thus able to directly test these modeling results. Over the course of a month, CRaTER samples the upstream solar wind as well as various regions of Earth's magnetotail. CRaTER data from multiple lunations demonstrate that Earth's magnetosphere at lunar distances produces no measurable influence on energetic particle flux, even at the lowest energies ( $>14 \mathrm{MeV}$ protons) where any effect should be maximized. For particles with energies of $14-30 \mathrm{MeV}$, we calculate an upper limit (determined by counting statistics) on the amount of shielding caused by the magnetosphere of $1.7 \%$. The high energy channel $(>500 \mathrm{MeV})$ provides an upper limit of $3.2 \%$. Citation: Case, A. W., H. E. Spence, M. J. Golightly, J. C. Kasper, J. B. Blake, J. E. Mazur, L. W. Townsend, and C. J. Zeitlin (2010), GCR access to the Moon as measured by the CRaTER instrument on LRO, Geophys. Res. Lett., 37, L19101, doi:10.1029/2010GL045118.

\section{Introduction}

\subsection{Magnetospheric Shielding}

[2] In the near-Earth environment the strong geomagnetic field provides shielding from energetic particles. The amount of shielding depends upon a variety of factors including the rigidity of the incident particles and the strength and orientation of the magnetic field in the region of interest [Størmer, 1955]. At small radial distances from Earth, a significant fraction of Galactic Cosmic Rays (GCR) can be blocked, but an investigation of the effectiveness of magnetospheric shielding in lunar orbit has not been pub-

\footnotetext{
${ }^{1}$ Center for Space Physics, Boston University, Boston, Massachusetts, USA.

${ }^{2}$ Department of Physics, University of New Hampshire, Durham, New Hampshire, USA. USA.

${ }^{3}$ Smithsonian Astrophysical Observatory, Cambridge, Massachusetts,

${ }^{4}$ The Aerospace Corporation, El Segundo, California, USA.

${ }^{5}$ The Aerospace Corporation, Chantilly, Virginia, USA.

${ }^{6}$ Department of Nuclear Engineering, University of Tennessee, Knoxville, Tennessee, USA.

${ }^{7}$ Southwest Research Institute, Boulder, Colorado, USA.

Copyright 2010 by the American Geophysical Union. 0094-8276/10/2010GL045118
}

lished before this study. However, previous studies have published data that are relevant to this investigation [e.g., Richardson, 2004]. Any significant blockage of energetic particles by the magnetosphere would be of interest to the manned spaceflight community, seeking to minimize exposure to harmful radiation in upcoming visits to the Moon.

[3] In this study, we refer to all energetic particles with energies greater than $10 \mathrm{MeV}$ as GCR. We recognize that some anomalous cosmic rays will also contribute to the signal that we term "GCR". Since this study is primarily concerned with the effects of particles with these energies, rather than their origins, we use this one term to describe all of the incident particles. Since this study was conducted during a time of very low solar activity, there was only one event in which solar particles contributed measurably to the energetic particle flux, and even then in an insignificant amount.

[4] Winglee and Harnett [2007] used a model of the magnetosphere to calculate the integrated perpendicular magnetic field $\left(B_{\perp}\right)$ along radial paths emanating from various lunar surface locations. The value of the integrated $B_{\perp}$ was used as a proxy for the shielding effectiveness from $1 \mathrm{GeV}$ protons. They found that significant shielding could be provided by the magnetosphere and the Earth itself, especially on the Earth-facing side of the Moon and during times of increased northward Interplanetary Magnetic Field (IMF).

[5] In a contrasting study that traced energetic particle trajectories through the magnetosphere, Huang et al. [2009] found that protons with energies greater than $10 \mathrm{MeV}$ were not significantly shielded by the Earth's magnetic field at lunar distances. In this paper, we use a relevant new data set to explore the aforementioned model discrepancy. We find that the magnetosphere at lunar distances does not provide shielding from GCR within the measured energy range. For the lowest energy protons that were measured, we calculate an upper limit of about $1.7 \%$ on the amount of GCR that may be shielded by the magnetosphere.

\subsection{CRaTER Instrument}

[6] The Cosmic Ray Telescope for the Effects of Radiation (CRaTER) [Spence et al., 2010] is an energetic particle telescope onboard the Lunar Reconnaissance Orbiter (LRO) [Tooley et al., 2010]. After launch on June 18th, 2009, LRO spent approximately 3 months in a commissioning orbit before entering its $50 \mathrm{~km}$ (average height above the surface) circular lunar polar orbit on September 15th, 2009. The orbital period of LRO is about 2 hours. LRO is a three-axis stabilized nadir-pointing spacecraft, with CRaTER mounted on one end of the spacecraft so as to have an unimpeded 
Table 1. Energy Deposits of Protons With Initial Energy, $\mathrm{E}_{\text {init }}^{\mathrm{a}}$

\begin{tabular}{lcccccc}
\hline $\mathrm{E}_{\text {init }}$ & $\mathrm{D} 1$ & $\mathrm{D} 2$ & $\mathrm{D} 3$ & $\mathrm{D} 4$ & $\mathrm{D} 5$ & $\mathrm{D} 6$ \\
\hline & & & $E_{\text {low }}$ & & & \\
14 & 1.87 & 4.71 & 0.00 & 0.00 & 0.00 & 0.00 \\
16 & 1.30 & 8.48 & 0.00 & 0.00 & 0.00 & 0.00 \\
18 & 1.05 & 11.50 & 0.00 & 0.00 & 0.00 & 0.00 \\
20 & 0.90 & 8.10 & 0.00 & 0.00 & 0.00 & 0.00 \\
22 & 0.79 & 6.47 & 0.00 & 0.00 & 0.00 & 0.00 \\
24 & 0.72 & 5.56 & 0.00 & 0.00 & 0.00 & 0.00 \\
26 & 0.66 & 4.94 & 0.00 & 0.00 & 0.00 & 0.00 \\
28 & 0.61 & 4.47 & 0.00 & 0.00 & 0.00 & 0.00 \\
30 & 0.57 & 4.11 & 0.00 & 0.00 & 0.00 & 0.00 \\
& & & & & & \\
& & & $E_{\text {high }}$ & & & \\
500 & $\mathbf{0 . 0 7}$ & 0.49 & $\mathbf{0 . 0 8}$ & 0.50 & $\mathbf{0 . 0 8}$ & 0.51 \\
750 & $\mathbf{0 . 0 6}$ & 0.42 & $\mathbf{0 . 0 6}$ & 0.42 & $\mathbf{0 . 0 6}$ & 0.42 \\
1000 & $\mathbf{0 . 0 6}$ & 0.38 & $\mathbf{0 . 0 6}$ & 0.39 & $\mathbf{0 . 0 6}$ & 0.39 \\
1500 & $\mathbf{0 . 0 5}$ & 0.35 & $\mathbf{0 . 0 5}$ & 0.35 & $\mathbf{0 . 0 5}$ & 0.35 \\
3000 & $\mathbf{0 . 0 5}$ & 0.33 & $\mathbf{0 . 0 5}$ & 0.33 & $\mathbf{0 . 0 5}$ & 0.33 \\
4000 & $\mathbf{0 . 0 5}$ & 0.33 & $\mathbf{0 . 0 5}$ & 0.33 & $\mathbf{0 . 0 5}$ & 0.33 \\
\hline
\end{tabular}

${ }^{\mathrm{a}} \mathrm{All}$ energies are in $\mathrm{MeV}$. Energies in boldface indicate that the energy is below the signal threshold for that particular detector.

view of both the lunar surface (nadir) and deep-space (zenith). The LRO spacecraft travels through all regions of the magnetosphere once a month, and since the LRO orbit is fixed in inertial space, the angle between the orbital plane and the Earth-Sun line goes through one full rotation each year. In this way, we sample all configurations of the LROEarth-Sun system every 3 months.

[7] CRaTER consists of a stack of six silicon detectors in thin/thick pairs separated by sections of Tissue Equivalent Plastic (TEP). Spence et al. [2010, Figure 5] show a schematic of the instrument layout. The thin detectors $(140 \mu \mathrm{m})$ are optimized for high energy deposits and the thick detectors $(1000 \mu \mathrm{m})$ are optimized for low energy deposits, and in particular, for protons. In nominal operating mode, an event is triggered when the energy deposit in any single detector rises above its threshold energy. A measurement is then made of the energy deposit in all six detectors. Directional information can be inferred for events that deposit energy into more than one detector, hereinafter called "detection coincidences." Endcaps at both ends of the instrument shield the detectors from protons with energies less than about $14 \mathrm{MeV}$. Extra mass placed around the edges of the detectors provides additional shielding from some particles which penetrate through the sides of the instrument.

\section{Data Preparation}

[8] Due to the fact that the published data products from the CRaTER instrument are in the form of linear energy transfer (LET) spectra, it is necessary to discuss here the production of higher-level data products used in this study. Specifically we use the measured LET values to infer the incident energy of the primary galactic cosmic ray protons.

[9] With particle transport codes we can simulate the passage of particles through the CRaTER shields, detectors, and TEP (collectively called the telescope). In this study, we use the slightly modified energy-loss routines described by Zeitlin et al. [1996]. The energy-loss calculation method was subsequently modified to include the density effect [Sternheimer et al., 1982], which is important at energies above several hundred $\mathrm{MeV} /$ nucleon. The code numerically integrates the energy-loss equation along the particle's path in $1-\mu \mathrm{m}$ steps to determine the amount of energy deposited into each detection element.

[10] We ran this code for protons with a range of incident energies in order to create a lookup table that contains the energy deposited into each telescope detection element as a function of the incident proton's initial energy, $\mathrm{E}_{\text {init }}$. Two ranges of energy deposits are shown in Table 1. The two delineated energy ranges $\left(14-30 \mathrm{MeV}\right.$ or $\mathrm{E}_{\text {low }}$ and $>500 \mathrm{MeV}$ or $\mathrm{E}_{\text {high }}$ ) are the energy channels produced for this investigation. If an energy deposit is below the threshold value for that detector, $\mathrm{E}_{\text {thresh }}$, the number is bolded in Table 1.

[11] Each energy channel is defined by a maximum and minimum energy deposit for each detector involved in the coincidence. Table 2 shows the energy deposit requirements for each detector and energy channel. If we do not expect to see an energy deposit above the signal threshold, $\mathrm{E}_{\text {thresh }}$, then the maximum allowed energy deposit for that channel is set to $\mathrm{E}_{\text {thresh }}$.

[12] If the expected range of proton energy deposits in a detector incorporates the Bragg peak of energy deposits, i.e., somewhere within our energy range are protons which will stop in that detector, then $\mathrm{E}_{\max }$ is set to the energy deposit of a stopping proton, $\mathrm{E}_{\text {stop }}=12.1 \mathrm{MeV}$ for a thick detector and 4.0 MeV for a thin detector. These maximum stopping energies were determined from the PSTAR range table database from the National Institute of Standards and Technology. The continuous-slowing-down approximation (CSDA) ranges were interpolated to find the energy of a proton that would stop in a detector. The data in this database are from International Commission on Radiation Units and Measurements [1993].

[13] It is worthwhile to note that the particle fluxes during the time-period of this investigation were low enough (compared to the signal processing capability of the CRaTER electronics) that accidental detection coincidences due to side-penetrating particles are inconsequential to our energy channel counting rates. We also note that data were used only from times when the CRaTER instrument was pointed within $1^{\circ}$ of nadir.

\section{Results}

[14] We now use the two CRaTER energy channels to investigate the magnetotail's influence on the flux of particles that fall within these channels. Figure 1 (top) shows the daily-averaged CRaTER summed singles rates for the thick detectors as black open circles. This rate includes all counts in D2, D4 or D6 with energies greater than about $14 \mathrm{MeV}$. Figure 1 (top) also shows the hourly averaged GCR flux from the Solar Isotope Spectrometer (SIS) instrument [Stone et al., 1998] on the Advanced Composition Explorer (ACE)

Table 2. Energy Deposit Requirements for Each Detector in an Energy Channel

\begin{tabular}{ccccccc}
\hline Limit & $\mathrm{D} 1$ & $\mathrm{D} 2$ & $\mathrm{D} 3$ & $\mathrm{D} 4$ & $\mathrm{D} 5$ & D6 \\
\hline $\mathrm{E}_{\text {low, } \min }$ & 0.57 & 4.11 & 0 & 0 & 0 & 0 \\
$\mathrm{E}_{\text {low, } \max }$ & 1.87 & $12.1^{\mathrm{a}}$ & $0.55^{\mathrm{b}}$ & $0.15^{\mathrm{b}}$ & $0.53^{\mathrm{b}}$ & $0.15^{\mathrm{b}}$ \\
$\mathrm{E}_{\text {high,min }}$ & 0 & 0.33 & 0 & 0.33 & 0 & 0.33 \\
$\mathrm{E}_{\text {high,min }}$ & $0.53^{\mathrm{b}}$ & 0.49 & $0.55^{\mathrm{b}}$ & 0.50 & $0.53^{\mathrm{b}}$ & 0.51 \\
\hline
\end{tabular}

${ }^{\mathrm{a}}$ Energy of a proton that stops in the detector.

${ }^{\mathrm{b}}$ Threshold energy of that detector. 


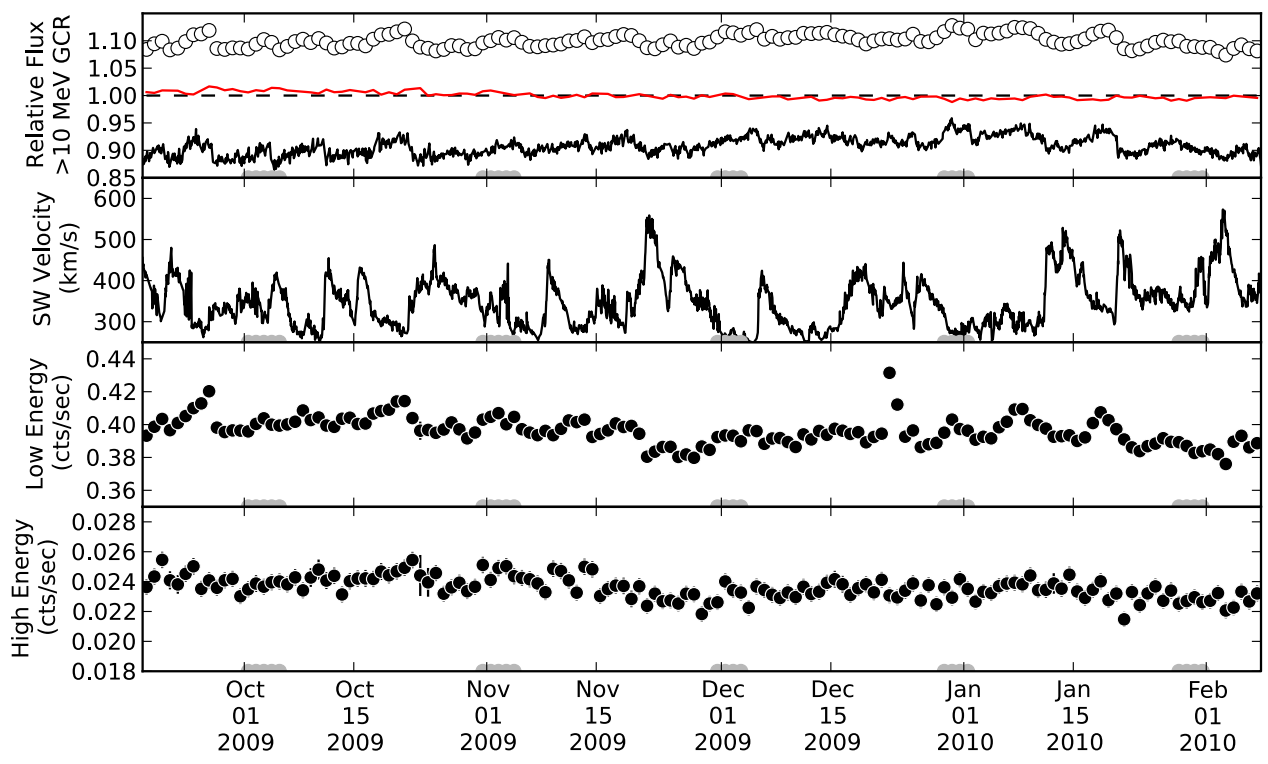

Figure 1. (top) CRaTER summed singles rates (black circles) and ACE $>10 \mathrm{MeV}$ GCR flux (thicker black line). These fluxes have been divided by their respective means and then offset vertically to improve clarity. The thin line (near 1.00) is the ratio of the CRaTER and ACE data that are shown in Figure 1 (top). The dashed line at 1.00 is to guide the eye. (top middle) Solar wind velocity from the SWEPAM instrument aboard ACE. (bottom middle and bottom) Time series of the two energy channelsof CRaTER that are defined in this paper. Gray regions on the x-axes indicate times when the Moon is within the magnetosphere.

spacecraft as a thicker black line. These data have been filtered to remove occasional noise spikes and calibrated to represent the flux of GCR with energies greater than $10 \mathrm{MeV}$ (R. Mewaldt, private communication, 2010). The ACE spacecraft is in orbit about the L1 point and is continuously in the solar wind and outside of any influence that the magnetosphere may have on energetic particles. Both of these data sets have been divided by their means and then offset vertically to improve clarity.

[15] Also shown in Figure 1 (top) is the ratio (solid red curve near 1.00) of the CRaTER and ACE/SIS data that are shown in the same panel. A black dashed line is shown at a value of 1 to guide the eye. Times when the Moon is within the magnetosphere are indicated by grey bars on the $\mathrm{x}$-axes. These times were determined by calculating an average magnetopause location from the model of Shue et al. [1998] as implemented by the GEOPACK collection of codes. The code was driven by typical solar wind conditions; $v_{s w}=400 \mathrm{~km} / \mathrm{s}, B_{z}=0$, and $n=5 \mathrm{~cm}^{-3}$, where $v_{s w}$ is the solar wind velocity, $B_{z}$ is the $\mathrm{z}$ component of the interplanetary magnetic field, and $n$ is the solar wind density.

[16] Figure 1 (top middle) shows the solar wind velocity as measured by the SWEPAM instrument on the ACE spacecraft [McComas et al., 1998]. Figures 1 (bottom middle) and 1 (bottom) show time series of the two energy channels over the course of five lunations. These are daily averages of CRaTER data from times when the instrument was in its nominal observing mode. Error bars are drawn for the singles rate (Figure 1, top) and the two energy channels (Figures 1, bottom middle and 1, bottom). These error bars represent the uncertainty due to counting statistics, and are smaller than the plot symbols for the singles rate and most of the points in the low energy rate. The high energy channel has an uncertainty of about $2-3 \%$. The larger uncertainty on October $23 \mathrm{rd}$ is due to a lower than usual amount of time being spent in nominal observing mode. The large jump in the flux of low energy particles around December 22, 2009 is believed to be from a very small solar particle event. Due to the low fluxes and energies associated with this event, it is not visible in the high energy or summed singles rate channels.

[17] As seen from Figure 1 (top), the CRaTER singles rates exhibit significant $(\sim 5 \%)$ temporal variations. All of these variations correspond qualitatively with those observed simultaneously by the ACE spacecraft whose data are taken far upstream of the magnetosphere and its possible influence. It can also be seen that many of the small depressions in the GCR flux are correlated with high-speed streams in the solar wind.

[18] The ratio of CRaTER to ACE/SIS flux (Figure 1, top) has variations of about $0.7 \%$ ( 1 standard deviation) when LRO is outside the magnetosphere. None of the passes through the magnetosphere show a significant drop in the flux that would indicate significant shielding by the magnetosphere. This indicates that the GCR flux at the CRaTER instrument (both inside and outside the magnetosphere) is undergoing the same fluctuations as the flux at ACE, which is at all times well outside the magnetosphere.

[19] We combine data from the five lunations shown in Figure 1 in order to quantify further any possible magnetotail shielding effect on GCR protons. Data from each energy channel are plotted in Figure 2 as a function of the angular location of the Moon in its orbit around the Earth. Figures 2 (top) and 2 (bottom) correspond to the $\mathrm{E}_{\text {low }}$ and $\mathrm{E}_{\text {high }}$ energy channels, respectively. Points indicate the mean of all of the daily measurements that were made within that angular location bin; error bars indicate 1 standard deviation of the distribution of the points that fell within that bin. In general, the error bars are on the order of $1-3 \%$. These uncertainties are dominated not by counting statistics, but by 


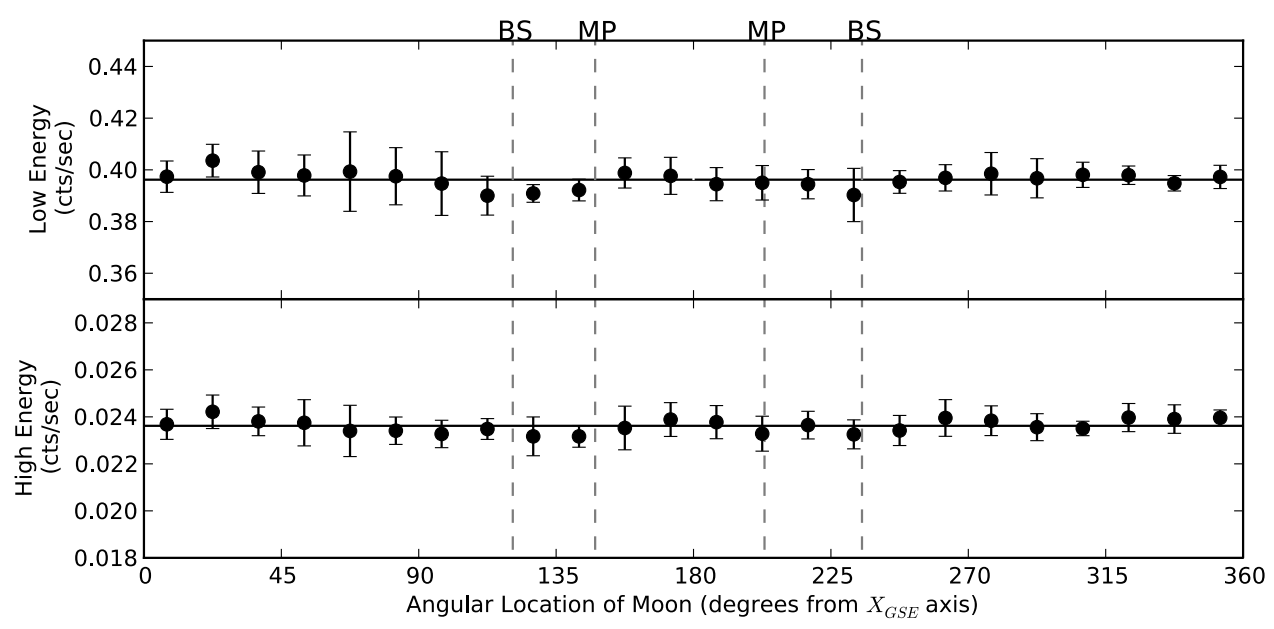

Figure 2. Flux in the 2 CRaTER derived energy channels as a function of the Moon's longitude in GSE coordinates around Earth. The inner pair of vertical dashed lines indicate the average modeled location of the magnetopause (MP) and the outer pair represent the modeled location of the bow shock (BS).

real variations in the GCR flux, predominantly driven by changes in the solar wind conditions.

[20] The inner set of vertical dashed lines represent the average location of the magnetopause (MP) and the outer set of vertical dashed lines represent the location of the bow shock (BS). The magnetopause locations are calculated as described above. The static bow shock model of Fairfield [1971] is used to calculate the angular location of the bow shock. This is a statistical empirical model derived from several hundred bow shock crossings. The specific coefficients used were for the meridional rotation without the $4^{\circ}$ rotation needed to correct for bow shock aberration due to Earth's orbital motion about the Sun, i.e., from the second column of Table 2 of Fairfield [1971].

[21] The average count rate is found for all times when the Moon is inside, $R_{i n}$, and outside, $R_{\text {out }}$, of the magnetosphere, and the percentage difference, $\mathrm{P}_{x}$, for each channel, $\mathrm{x}$, is calculated as:

$$
P_{x}=\frac{\left(R_{\text {in }, x}-R_{\text {out } x}\right)}{\left(R_{\text {in }, x}+R_{\text {out }, x}\right) / 2} \times 100 \%
$$

Therefore, a positive (negative) $P_{x}$ indicates a relative over (under-)abundance of GCR within the magnetosphere. The calculated values for $\mathrm{P}_{l o w}$ and $\mathrm{P}_{h i g h}$ are $+0.07 \% \pm 1.7 \%$ and $+0.33 \% \pm 3.2 \%$, respectively. $P_{x}$ values are also calculated for when the Moon is in the sheath region (inside the bow shock, but not inside the magnetopause), the values are $\mathrm{P}_{\text {low, sheath }}=-0.8 \% \pm 1.5 \%$ and $\mathrm{P}_{\text {high,sheath }}=-1.4 \% \pm$ $2.9 \%$. The uncertainties on the $\mathrm{P}_{x}$ values are the mean of the uncertainties of all points that were inside that region of interest (i.e., for the magnetospheric values, uncertainties from all of the points from inside the magnetosphere were used).

\section{Conclusions}

[22] We have outlined a method by which we define energy channels in CRaTER data through model-informed responses to incident particle species and energies. We use this method to define energy channels representing incident protons of $14-30 \mathrm{MeV}$ and $>500 \mathrm{MeV}$. Rates in these channels, organized as a function of lunar orbital position relative to magnetosphere location are then used to investigate the magnetosphere's shielding efficiency for comparison with earlier model predictions.

[23] Neither of the energy channels exhibit any significant decrease in the flux of GCR when the Moon is within the Earth's magnetotail. The lowest energy channel should be most affected by any magnetotail shielding. In this channel we see a $0.07 \% \pm 1.7 \%$ decrease in flux when the Moon is within the magnetotail. The statistical uncertainty of the mean of $1.7 \%$ provides an upper limit for the amount of low energy GCR that may be shielded when the Moon is in the magnetotail. The data from the high energy channel constrain the shielding effect to no more than $3.2 \%$. In addition, there is no evidence for shielding in either energy channel on any of the five individual magnetotail passes included in the statistical ensemble.

[24] The majority of the uncertainty in our analysis comes from real variations in the cosmic ray flux as a result of changing solar wind and interplanetary magnetic field conditions. As seen in Figure 1, high-speed solar wind streams are associated with many of the cosmic ray decreases seen in both the ACE/SIS and the CRaTER data. Other studies have also reported this phenomenon [e.g., Richardson et al., 1996, and references therein]. Future studies may investigate further the causes of these variations, but we have not attempted to account for them in this analysis.

[25] Previously published data also support the conclusions drawn from the current study. For example, Richardson [2004, Figure 7] show data from the Helios-1 and Helios-2 spacecraft, which were well outside of the magnetosphere in a solar orbit, and the IMP-8 spacecraft, which spent a portion of its orbit inside the magnetosphere. The GCR rates are shown for all three spacecraft, and qualitatively the GCR count rates from all of them agree well, whether IMP-8 was within the magnetosphere or not.

[26] The observations made in the current study are in agreement with the modeling results of Huang et al. [2009], which traced isotropic distributions of GCR with energies of 1,10 , and $100 \mathrm{MeV}$ through an empirical model magnetosphere. The authors determined that within their statistical 
uncertainty of about $2 \%$, there was no shielding of GCR by the magnetosphere.

[27] The conclusions of Huang et al. [2009] are in stark contrast to results from other recent modeling efforts by Winglee and Harnett [2007] that used an MHD model of the magnetosphere to infer the amount of GCR that would be shielded by the magnetosphere at various places on the lunar surface. This study concentrated on energetic particles that are incident from specific directions, while neglecting to factor in the isotropic nature of the incident radiation. While it is possible that the trajectories outlined by Winglee and Harnett [2007] are occurring, it is just as likely that other particles that would not have impinged on the lunar surface are scattered into trajectories which do impact the Moon. The result being that there is no net shielding of GCR in the magnetotail. Winglee and Harnett [2007] do not quantify the percentage of GCR that would be shielded, but presumably the high end of the colorbar for Figures 2 and 3 of Winglee and Harnett [2007] indicates that they expect a very significant amount, say more than $50 \%$, of the GCR to be shielded from many locations on the Moon.

[28] It should be noted that the daily averaging used in this investigation does not allow us to investigate magnetospheric shielding processes with respect to location around the Moon. Winglee and Harnett [2007] predicted greater shielding on the equatorial Earth-facing side of the Moon, as compared to higher latitudes and farside locations. We did not sort our data according to solar wind or interplanetary magnetic field (IMF) conditions, which were also predicted by Winglee and Harnett [2007] to have some influence on the shielding efficiency of the magnetotail, though future studies may investigate this possibility.

[29] Acknowledgments. We would like to thank Chia-Lin Huang for helpful discussions, Dick Mewaldt and the SIS instrument team for providing GCR data from the ACE spacecraft, and the ACE SWEPAM team and the ACE Science Center for providing the ACE solar wind data. This work was supported by the LRO/CRaTER project under contract NASA NNG05EB92C.

\section{References}

Fairfield, D. H. (1971), Average and unusual locations of the Earth's magnetopause and bow shock, J. Geophys. Res., 76, 6700-6716, doi:10.1029/JA076i028p06700.

Huang, C.-L., H. E. Spence, and B. T. Kress (2009), Assessing access of galactic cosmic rays at Moon's orbit, Geophys. Res. Lett., 36, L09109, doi:10.1029/2009GL037916.
International Commission on Radiation Units and Measurements (1993), Stopping powers and ranges for protons and alpha particles, Rep. 49, Int. Comm. on Radiat. Units and Meas., Bethesda, Md.

McComas, D. J., S. J. Bame, P. Barker, W. C. Feldman, J. L. Phillips, P. Riley, and J. W. Griffee (1998), Solar Wind Electron Proton Alpha Monitor (SWEPAM) for the Advanced Composition Explorer, Space Sci. Rev., 86, 563-612, doi:10.1023/A:1005040232597.

Richardson, I. G. (2004), Energetic particles and corotating interaction regions in the solar wind, Space Sci. Rev., 111, 267-376, doi:10.1023/ B:SPAC.0000032689.52830.3.

Richardson, I. G., G. Wibberenz, and H. V. Cane (1996), The relationship between recurring cosmic ray depressions and corotating solar wind streams at $\leq 1$ AU: IMP 8 and Helios 1 and 2 anticoincidence guard rate observations, J. Geophys. Res., 101, 13,483-13,496, doi:10.1029/ 96JA00547.

Shue, J.-H., et al. (1998), Magnetopause location under extreme solar wind conditions, J. Geophys. Res., 103, 17,691-17,700, doi:10.1029/ 98JA01103.

Spence, H. E., et al. (2010), CRaTER: The Cosmic Ray Telescope for the Effects of Radiation experiment on the Lunar Reconnaissance Orbiter mission, Space Sci. Rev., 150, 243-284, doi:10.1007/s11214-0099584-8.

Sternheimer, R. M., S. M. Seltzer, and M. J. Berger (1982), Density effect for the ionization loss of charged particles in various substances, Phys. Rev. B, 26(11), 6067-6076, doi:10.1103/PhysRevB.26.6067.

Stone, E. C., et al. (1998), The Solar Isotope Spectrometer for the Advanced Composition Explorer, Space Sci. Rev., 86, 357-408, doi:10.1023/A:1005027929871.

Størmer, C. M. (1955), The Polar Aurora, Clarendon, Oxford, U. K.

Tooley, C. R., M. B. Houghton, R. S. Saylor, C. Peddie, D. F. Everett, C. L. Baker, and K. N. Safdie (2010), Lunar Reconnaissance Orbiter mission and spacecraft design, Space Sci. Rev., 150, 23-62, doi:10.1007/s11214009-9624-4

Winglee, R. M., and E. M. Harnett (2007), Radiation mitigation at the Moon by the terrestrial magnetosphere, Geophys. Res. Lett., 34, L21103, doi:10.1029/2007GL030507.

Zeitlin, C., L. Heilbronn, J. Miller, W. Schimmerling, L. W. Townsend, R. K. Tripathi, and J. W. Wilson (1996), The fragmentation of $510 \mathrm{mev} /$ nucleon iron-56 in polyethylene. II. Comparisons between data and a model, Radiat. Res., 145(6), 666-672, doi:10.2307/3579356.

J. B. Blake, The Aerospace Corporation, 2310 E. El Segundo Blvd., El Segundo, CA 90245, USA.

A. W. Case, Center for Space Physics, Boston University, 725 Commonwealth Ave., Boston, MA 02215, USA. (tonycase@bu.edu) M. J. Golightly and H. E. Spence, Department of Physics, University of New Hampshire, DeMeritt Hall, 9 Library Way, Durham, NH 03824, USA.

J. C. Kasper, Smithsonian Astrophysical Observatory, 60 Garden St., Cambridge, MA 02138, USA.

J. E. Mazur, The Aerospace Corporation, 15049 Conference Center Dr. Ste. 600, Chantilly, VA 20151, USA.

L. W. Townsend, Department of Nuclear Engineering, University of Tennessee, 315 Pasqua Engineering Bldg., Knoxville, TN 37996, USA.

C. J. Zeitlin, Southwest Research Institute, 1050 Walnut St., Ste. 300, Boulder, CO 80302, USA. 